\title{
Uplink Transmission Timing in WCDMA
}

Fredrik Gunnarsson, David Törnqvist, Erik Geijer Lundin

Gunnar Bark, Niclas Wiberg, Eva Englund

\author{
Division of Communication Systems \\ Department of Electrical Engineering \\ Linköpings universitet, SE-581 83 Linköping, Sweden \\ WWW: http://www. control.isy.liu.se \\ E-mail: fred@isy.liu.se, tornqvist@isy.liu.se \\ geijer@isy.liu.se
}

22nd September 2003

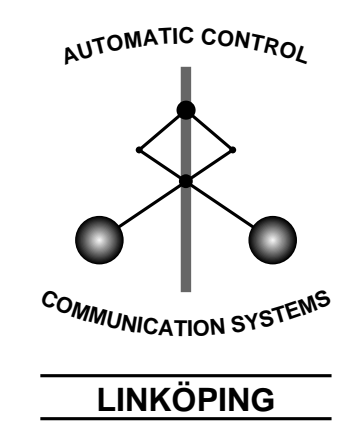

Report no.: LiTH-ISY-R-2546

Submitted to VTC'03 Fall, Orlando, FL, USA

Technical reports from the Control \& Communication group in Linköping are available at http://www.control.isy.liu.se/publications. 


\begin{abstract}
In wireless network uplink communications, there is a tradeoff between transmission coordination to avoid overload situations, and distributed transmission decisions to adapt to fast channel variations. Here, uplink transmission timing (UTT) is proposed as a scheme to allow some load control support, while transmitting mainly when the channel is favorable. It utilizes channel state feedback in the form of power control commands, which already are available in the system. Simulations illustrate the transmission timing behavior, and also indicate that UTT is a power and intercell interference efficient scheme to transport data compared to traditional dedicated channels with continuous transmissions and to schemes where transmission decisions are random.
\end{abstract}

\title{
Keywords:
}




\title{
Uplink Transmission Timing in WCDMA
}

\author{
Fredrik Gunnarsson*†, David Törnqvist* Erik Geijer-Lundin* \\ Gunnar Bark $^{\dagger}$, Niclas Wiberg ${ }^{\dagger}$ and Eva Englund ${ }^{\dagger}$ \\ ${ }^{*}$ Div. of Control and Communication, Linköping University, SE-581 83 LINKÖPING, SWEDEN, \\ Email: $\{$ fred, tornqvist,geijer $\}$ isy.liu.se \\ ${ }^{\dagger}$ Ericsson Research, P.O. Box 1248, SE-581 12 LINKÖPING, SWEDEN, \\ Email: \{fredrik.gunnarsson, gunnar.bark, niclas.wiberg, eva.englund\}@ericsson.com
}

\begin{abstract}
In wireless network uplink communications, there is a tradeoff between transmission coordination to avoid overload situations, and distributed transmission decisions to adapt to fast channel variations. Here, uplink transmission timing (UTT) is proposed as a scheme to allow some load control support, while transmitting mainly when the channel is favorable. It utilizes channel state feedback in the form of power control commands, which already are available in the system. Simulations illustrate the transmission timing behavior, and also indicate that UTT is a power and inter-cell interference efficient scheme to transport data compared to traditional dedicated channels with continuous transmissions and to schemes where transmission decisions are random.
\end{abstract}

\section{INTRODUCTION}

With limited availability of radio resources and an increasing interest in wireless data services, it is of utmost importance to utilize the resources efficiently. Recently, there has been a strong focus towards efficient downlink (from base stations to terminals) radio resource utilization in $3 \mathrm{G}$ systems. The improvements significantly enhance both application and system performance. Much of the gain is due to time-sharing of base station radio resources among users and more resource control distributed to the base station. The uplink (from terminals to base stations) situation is different, since resource coordination like time-sharing requires extensive signaling. The objectives with an enhanced uplink can therefore be flexible and efficient data transmission with minimal centralized coordination.

One possible realization of a data service in WCDMA is over a dedicated radio bearer [1], [2], with power control to mitigate fast fading. To maintain acceptable service quality in the uplink, this means a high mobile transmitter power when the channel is infavorable, and vice versa. Therefore, it is less efficient to transmit data during faded channel states. These periods also result in significantly higher interference to other base stations. Hence for delay insensitive services, it can be motivated to avoid transmitting during infavorable channel states to reduce the battery consumption, and to limit the inter-cell interference.

On the lowest uplink layer, the terminal sends a waveform according to selected coding and modulation over the established link to the base station(s). The channel quality is time-varying, and the link is best utilized by adapting coding and modulation to the channel state [3]. Several schemes are proposed to as tightly as possible meet the Shannon channel capacity, e.g. the water filling in [4]. However, in practice channel state information has to be fed back from the receiver to the transmitter, and this critically limits the performance [5].

In wireless networks, where the main source of disturbance originates from other connections, the situation is more delicate. Maximizing the resource utilization is not only a matter of optimizing the channel utilization of the individual links, but also to cater for the mutual interference between connections. Furthermore, to support mobility from one base station to another, much coordination is centralized to the radio network controllers (RNC's). This facilitates handover, but delays decisions which effect individual radio links. Uplink transmitted power control is based on information fed back from the receiver, in this case the base station. A natural design choice is therefore to adapt coding and modulation only seldom, but to update transmitter power often to compensate for channel variations. This means that the resource sharing is managed by the RNC, and distributed power control realizes and maintains the resource sharing.

In the downlink, the total base station power essentially limits the available resource. The network is planned and deployed to provide coverage and quality of service to users also when all surrounding base stations are operating at maximum power levels. The uplink is different, since the unpredictable interference from users in surrounding cells may limit coverage, capacity and quality of service [6]. The main uplink load quantity at a base station is the uplink received power relative to the noise - the uplink noise rise [7]. This quantity is difficult to predict in time, since it directly depends on connections in surrounding cells. Moreover, it is difficult to accurately measure, and related estimates are necessary [8]. If the resource sharing is coordinated from the RNC, only primarily slow mechanisms like admission control are plausible.

On the other hand, if the channel variations are relatively slow, centralized detailed management of the users' power and data rate is possible. In the downlink, if it is possible to utilize high order modulation to benefit from high powers to individual users, it is most efficient to fully time-share the downlink channel among users at every base station [9]. This is not as natural in the uplink because the resource utilization is more difficult to predict, and it is less likely that an individual user can use up all the available uplink capacity in a base station. Several users in each cell have to transmit simultaneously to fully utilize the uplink. A detailed timecoordination of the uplink requires substantial overhead sig- 
naling where all terminals must indicate their available power and buffer status and keep a pilot signal for channel estimation. In addition, to completely coordinate uplink transmissions in a cellular system, coordination between base stations is needed. This involves the RNC's and thereby additional signaling and delays. The consequence is a reduced risk of uplink overload, but increased signaling overhead and reduced possibilities to adapt to channel variations [10]-[13]. Alternative approaches are to coordinate transmissions only within each base station or to rely on pure statistical multiplexing, where the decision to transmit is distributed to the terminals [14]-[17]. Advantages of such schemes are that the terminal can quickly adapt to variations in traffic demand, reducing initial delays, and adapt to rapid variations in channel quality. However, no or limited network coordination means that the there is a risk of uplink overload when more users than the network can support transmit simultaneously.

This paper propose uplink transmission timing, which is a scheme for uplink data transmission over dedicated wireless channels. It uses power control information to distributedly determine whether it is favorable to transmit or not. Some centralized control is also included to allow control over the uplink load. Section II describes the notation, models and algorithms that are central in the paper. Uplink transmission timing is introduced in Section III to utilize differential channel state feedback in the form of power control commands and to aim at transmitting when the channel is favorable relative the channel situation over a longer time frame. Section IV provides illustrative and comparative simulations, and Section V some concluding remarks.

\section{Dedicated Channels In WCDMA}

\section{A. Dedicated Channels}

The focus here is on services, which are allocated resources dedicated to a specific mobile. There are two types of dedicated physical channels in WCDMA - Dedicated Physical Data Channel (DPDCH) and Dedicated Physical Control Channel (DPCCH). The former carries data and control information from higher layers, whereas the latter carries physical control information and is therefore always present. In the uplink these two physical channels are I/Q multiplexed and transmitted in parallel (as opposed to the downlink with timemultiplexed dedicated physical channels). The physical layer offers information transfer services to higher layers, and the transport channel dedicated to a specific mobile is the Dedicated Channel (DCH). The medium access control (MAC) layer provides data transfer services on logical channels for control information (e.g. Dedicated Control Channel, DCCH) and user data (e.g. Dedicated Traffic Channel, DTCH). Both $\mathrm{DCCH}$ and DTCH can be mapped onto DCH.

A specific service is thus realized as one or several traffic channels (DTCH's) and supported by one or several control channels (DCCH's). In a multi-service situation (for example speech and web-browsing), some DTCH's transfer the delaysensitive services, while others transfer services less sensitive to delays.
The data units (data blocks) transferred over the dedicated transport channels occupy at most the transmission time interval (TTI), which is $10,20,40$ or $80 \mathrm{~ms}$. These are either received correctly or erroneously (block errors). For further information on dedicated channels, see for example [1] and references. During TTI's when there are no data units to transmit over the DTCH's and DCCH's, there is no need to transmit over DPDCH at all. This is referred to as discontinuous transmission, DTX.

\section{B. Wireless Networks and Power Control}

Some quantities will be expressed both in linear and logarithmic scale $(\mathrm{dB})$. Linear scale is indicated by the bar notation $\bar{g}(t)$. By neglecting data symbol level effects, the communication channel can be seen as a time varying power gain $\bar{g}(t)$. It is instructive to separate the power gain into a slowly varying $\bar{g}^{a v}(t)$ (essentially path loss and shadow fading at low to moderate speeds) and a rapidly varying component $\bar{g}^{f}(t)$ (fast fading) such that $\bar{g}(t)=\bar{g}^{a v}(t) \bar{g}^{f}(t)$ [18]. The power gain from mobile $i, i=1, \ldots, M$ to base station $j, j=1, \ldots, B$ is denoted $\bar{g}_{i j}(t)$. If the transmitter power of mobile $i$ is $\bar{p}_{i}(t)$ and the connected base station $j_{i}$ is interfered from other connections and thermal noise by the power $\bar{I}_{i}(t)$, the signal-to-interference ratio $(S I R)$ at the receiver is given by

$$
\bar{\gamma}_{i}(t)=\frac{\bar{p}_{i}(t) \bar{g}_{i j_{i}}(t)}{\bar{I}_{i}(t)}=\frac{\bar{g}_{i j_{i}}(t) \bar{p}_{i}(t)}{\sum_{k \neq i} \bar{g}_{i j_{k}}(t) \bar{p}_{k}(t)+\bar{\nu}_{i}(t)},
$$

where $\bar{\nu}_{i}(t)$ is thermal noise at receiver. In logarithmic scale, the SIR expression becomes

$$
\gamma_{i}(t)=p_{i}(t)+g_{i j_{i}}(t)-I_{i}(t)
$$

In the uplink, the connections are separated by codecorrelation. This only works if all connections are received with a SIR, that is motivated by the associated data rate. Therefore, power control is an important means to prevent mobiles close to the base station to be received with much better SIR than more distant mobiles. The QoS requirements can approximately be associated to a desired block error rate, $B L E R$, which in turn can be related to a required target SIR level, $\bar{\gamma}_{i}^{t}(t)$. To ensure that the desired BLER is met, target SIR is regularly re-assigned based on block error statistics. This is referred to as outer loop power control. The distributed power control algorithms are based on local feedback information, typically to meet $\operatorname{SIR}, \bar{\gamma}_{i}(t) \approx \bar{\gamma}_{i}^{t}(t)$, despite time-varying channels. The inner loop power control [19] operates at $1500 \mathrm{~Hz}$ and is faster than the outer loop. The power level is increased/decreased depending whether the measured SIR is below or above target SIR, and implemented as:

$$
\begin{array}{rc}
\text { Receiver : } & e_{i}(t)=\gamma_{i}^{t}(t)-\gamma_{i}(t) \\
& s_{i}(t)=\operatorname{sign}\left(e_{i}(t)\right) \\
\text { Transmitter : } & p_{T P C, i}(t)=\Delta_{i} s_{i}(t) \\
& p_{i}(t+1)=p_{i}(t)+p_{T P C, i}(t)
\end{array}
$$

where $\Delta_{i}$ is the step size. The base station feeds back the power control commands $s_{i}(t)$ to the mobile using the 
downlink DPCCH. Then, the mobile updates the transmitter power $p_{i}(t)$ based on the demodulated and decoded power control commands. This scheme gives acceptable SIR provided that the channel variations are not too fast, and that the system is not overloaded [20]. In WCDMA, inner loop power control is applied to DPCCH, which is continuously transmitted. The $\mathrm{DPDCH}$ power is obtained as

$$
p_{D P D C H}=\beta \cdot p_{D P C C H},
$$

where $\beta$ is a configurable power offset which depend on the amount of data transmitted over DPDCH [19].

If power control is perfect $\left(\gamma_{i}(t)=\gamma_{i}^{t}(t)\right)$, the mobile transmission power is given by (2)

$$
p_{i}(t)=\underbrace{\left(\gamma_{i}^{t}(t)+I_{i}(t)-g_{i j_{i}}^{a v}(t)\right)}_{p_{i}^{a v}(t)}+\underbrace{\left(-g_{i j_{i}}^{f}(t)\right)}_{p_{i}^{f}(t)},
$$

where the notation of local average power $p_{i}^{a v}(t)$ (slowly varying) and rapidly varying power $p_{i}^{f}(t)$ is intuitive if assuming that the power variations are mainly due to power gain variations. The fast fading is characterized by deep fades, meaning that temporary high power levels are needed to fully compensate for such fades.

\section{UPLINK TRANSMISSION TIMING}

When the uplink load increases, an alternative to disconnect users to maintain system stability is to use flexible services, which reduces the uplink activity on demand. One such approach could be to limit the time the mobiles transmit by assigning an activity percentage (activity factor $A_{f}$ ) to the mobiles, which transmits at random to meet this activity. The activity factor allows some slow RNC control of the uplink load. At TTI $t$, the mobile is therefore either active $A(t)=1$ or inactive $A(t)=0$, such that $\mathrm{E}\{A(t)\}=A_{f}$. The uplink load contribution from the particular mobile is thus directly related to the activity factor. However, there is no correlation between transmission instants and when the channel is favorable. As discussed previously, it is highly interesting to avoid transmitting during deep fades.

The main idea behind uplink transmission timing (UTT) is to transmit discontinuously to meet an assigned activity factor, but to utilize information about the transmission power to select the transmission instants carefully to avoid transmitting during fading dips. Since fading information is reflected in the power level, a plausible solution could be to transmit only when the power level is less than a threshold $h$. The fast power gain variations, and consequently also the varying transmission powers, are typically independent between different mobiles. Therefore, transmission decisions based on power level thresholding gives good statistical multiplexing properties. If the average power $p_{i}^{a v}(t)$ would be constant, and the rapidly varying power $p_{i}^{f}(t)$ stationary, there is a direct link between the threshold, the activity factor $A_{f}$ and the cumulative distribution function (CDF) of the rapidly varying power. The threshold $h$ is implicitly given by

$$
A_{f}(h)=\operatorname{CDF}\left(p_{i}^{f}\right)=\mathrm{P}\left(p_{i}^{f}<h\right) .
$$

In practice, the average power is not constant, and the varying power not stationary, partly due to a varying velocity. Furthermore, not all data transport can be discontinued. The physical control channel DPCCH is always transmitted, and delay-sensitive control and data on DCCH's and DTCH's should neither be discontinued.

Essentially, the proposed uplink transmission timing estimates the current activity $A_{f}^{\text {est }}(t)$ using recent transmission history $A(\tau), \tau \leq t$ and adjusts the threshold $h$ so that $A_{f}^{e s t}(t) \approx A_{f}$. Algorithm 1 provides the detailed steps. In this context, fully transmit is equivalent to transmitting all channels including less delay-sensitive information, whereas not fully transmit means that only delay-sensitive control information and data are transmitted.

\section{Algorithm 1 (Uplink Transmission Timing)}

(i) Monitor the transmission power $p(t)$ (for example by monitoring the cumulative sum of power updates $p_{T P C}(t)$ in $\left.(3 \mathrm{c})\right)$

(ii) Fully transmit during TTI $t$ if the transmission power $p(t)$ is lower than a threshold $h(t)$. The activity $A(t)=1$ if fully transmitting during TTI $t$, and 0 otherwise.

(iii) Estimate the current activity $A_{f}^{e s t}(t)$ using recent transmission history $A(\tau), \tau \leq t$. An applicable and memory-efficient implementation is an exponential filter

$$
A_{f}^{e s t}(t)=d A_{f}^{e s t}(t-1)+(1-d) A(t)
$$

(iv) Adapt the threshold $h(t)$ if the current activity estimate $A_{f}^{e s t}(t)$ is different from the desired activity $A_{f}$. This can be implemented as an integrating control function

$$
h(t+1)=h(t)+b\left(A_{f}-A_{f}^{e s t}(t)\right) .
$$

Uplink transmission timing can thus be seen as a feedback system for threshold control and transmission decision. The block diagram in Figure 1 describes the dynamics of UTT with the choices of activity estimation and threshold control schemes used in simulations in Section IV.

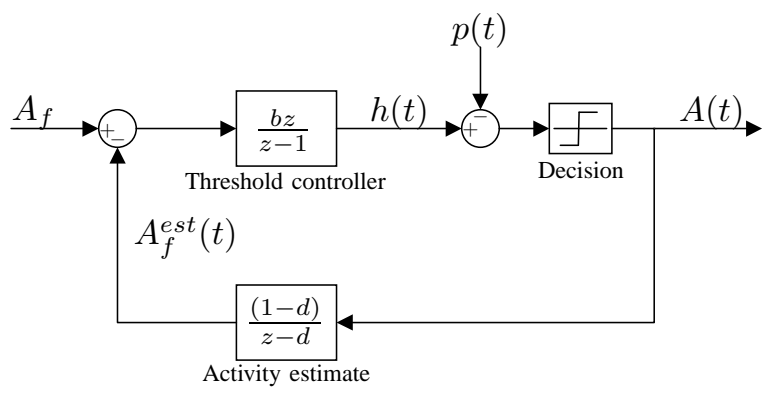

Fig. 1. Block diagram describing the dynamics of uplink transmission timing 


\section{Simulations}

Uplink transmission timing is characterized and compared to employing random transmission decisions in simulations.

\section{A. Simulation Environment}

The simulated scenarios are sector cell wireless networks with wrap around. Only path loss (exponent $\alpha=3.5$ ) and fast fading (ITU Pedestrian A and 3GPP Typical Urban) are included in the power gain model, since low to moderate velocities are considered, and the shadow fading variations are then fully compensated for by power control. The focus is primarily on the comparative behavior of UTT and not on the resulting capacity differences. Radio bearers for $64 \mathrm{kbps}$ are parameterized according to the test configurations in [2], and $128 \mathrm{kbps}$ are realized based on the former, but with appropriate power offset adjustments.

\section{B. Illustrative Example}

Figure 2 illustrates the behavior of UTT. Clearly, bad channel states are avoided to a great extent. The activity $A_{f}^{e s t}$ is estimated by lowpass filtering the activity $A(t)$ and used to adjust the threshold $h(t)$. Note the oscillatory behavior of the activity.

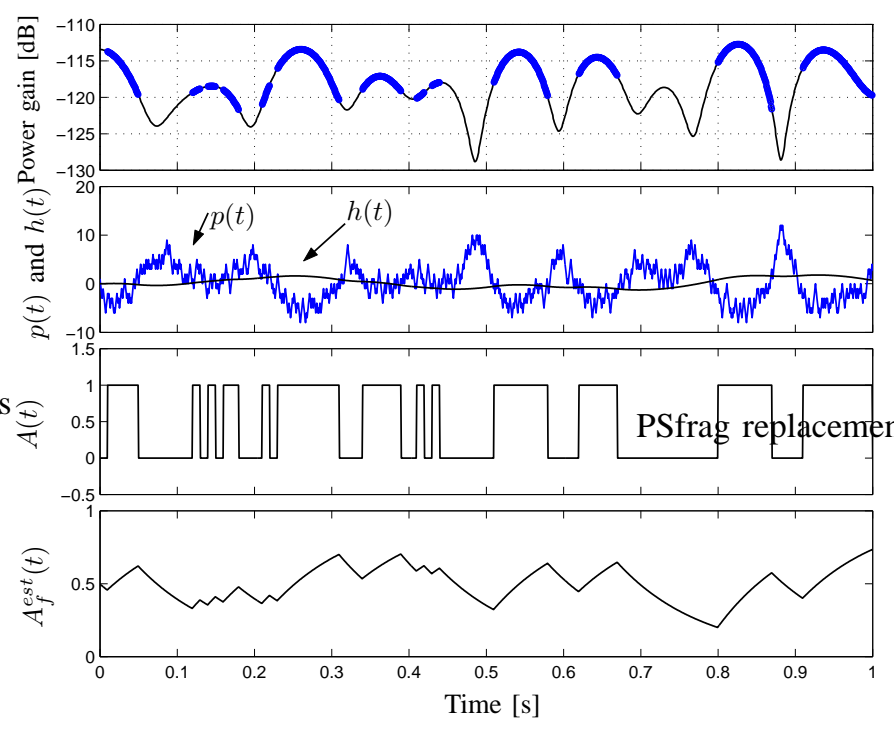

Fig. 2. Uplink transmission timing example.

\section{Single User Behavior}

Naturally, the behavior and adaptability of UTT depend on the parameters $b$ and $d$. However, extensive simulations to analyze the required transmitter power indicate that the dependency is not critical. However, a sufficiently large $d$ is needed to obtain adequate activity averaging, and the parameters $d=0.994$ and $b=0.08$ are used in the simulations.

The performance depends on the mobile velocity, or more specifically on the relation between the velocity and the TTI length in slots. A shorter TTI gives a better resolution in the transmission decision. Figure 3 depicts the average power reductions compared to random transmission for two different velocities. In the sequel, the current 15 slots per TTI is used with mobile velocities $3 \mathrm{~km} / \mathrm{h}$ to deemphasize the TTI dependency. For activity factor $A_{f}=0.5$ and $128 \mathrm{kbps}$, the
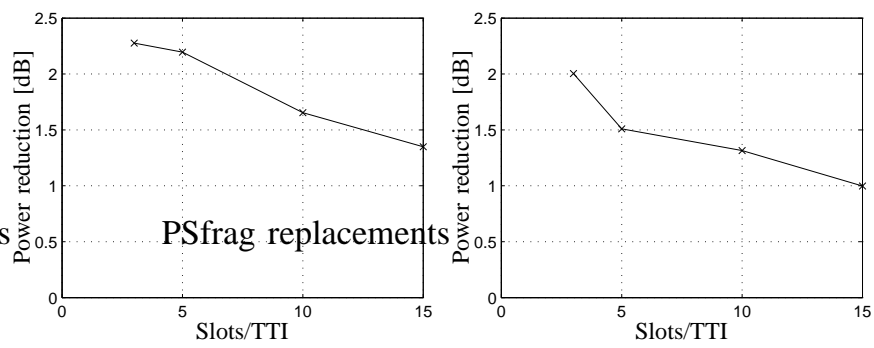

Fig. 3. Average power reduction at different TTI-lengths when using UTT instead of random transmission decisions. Velocity: $3 \mathrm{~km} / \mathrm{h}$ (left) and $20 \mathrm{~km} / \mathrm{h}$ (right).

average reduction compared to random is $1.7 \mathrm{~dB}(\mathrm{Ped} \mathrm{A})$ and $0.7 \mathrm{~dB}$ (TU). This radio bearer has the same mean data rate as $64 \mathrm{kbps}$ with continuous activity. A comparison of UTT and the latter gives an average power reduction of $1.6 \mathrm{~dB}(\mathrm{Ped} \mathrm{A})$ and $0.6 \mathrm{~dB}(\mathrm{TU})$

The same conclusion holds for the inter-cell interference contribution from a specific mobile as seen in Figure 4. This is natural since according to (1) this contribution $\bar{p}_{k}(t) \bar{g}_{i j_{k}}(t)$ depends on the transmission power and the power gain, and the latter has the same distribution for both UTT and random transmission.

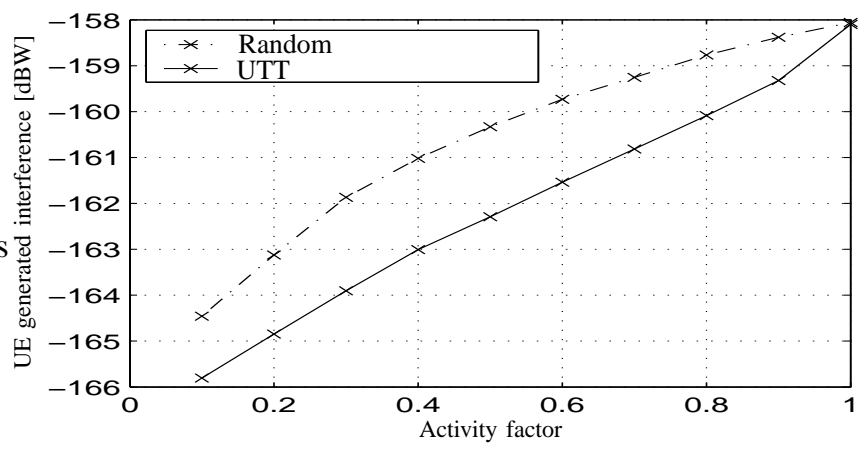

Fig. 4. Comparison between UTT and random transmission in terms of intercell interference contribution at a different cell than the user is connected to.

The control channel power overhead is almost negligible when the activity is high, but most noticeable at low activity. It is therefore instructive to study the power efficiency in terms of power per kbps for the two schemes and different activity factors. Figure 5 reveals that the optimal activity is different for $64 \mathrm{kbps}$ and $128 \mathrm{kbps}$, since the control channel overhead is different. Furthermore, it is more power efficient to use the dedicated channels with UTT than with full activity, and it is more efficient to realize a $64 \mathrm{kbps}$ service as a $128 \mathrm{kbps}$ dedicated channel with activity factor 0.5.

With the same frequency in all base stations, there is a risk of corner effects, where a mobiles suddenly gets much better conditions to a new base station, and before (soft) handover to the new cell is completed the mobile critically 

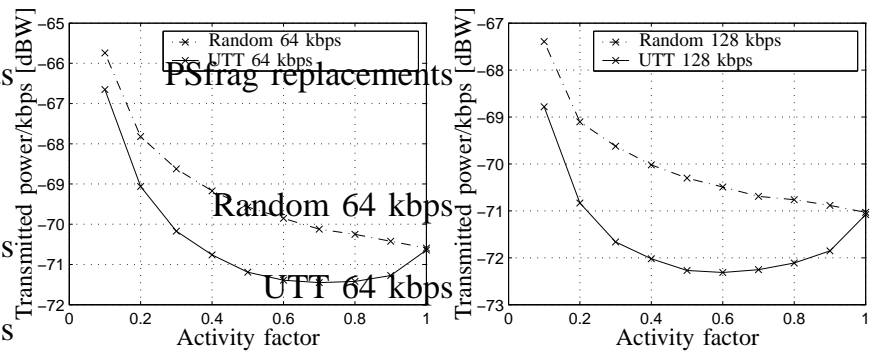

Fig. 5. Power effi ciency in terms of transmitted power per kbps for different activity factors.

disturb all other links at this base station. However with UTT, the power increase to compensate for the abrupt power gain change discontinues transmission for some time, until the threshold has reached the new power level as seen in Figure 6. Hence, there is time to complete the handover, start controlling the power relative the new base station, and the critical interference is avoided.
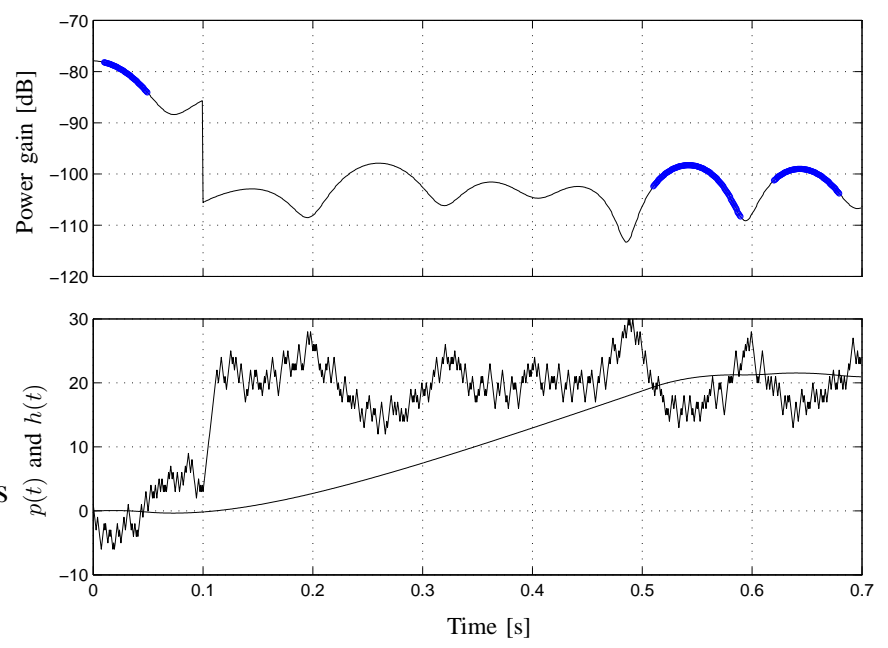

Fig. 6. UTT and an abrupt change in channel gain. Top: Power gain (thick sections indicate data transmission). Bottom: Uplink power $p(t)$ and threshold $h(t)$.

\section{CONCLuSions}

Schemes for full coordination of the WCDMA uplink suffers from extensive signaling, whereas no coordination means a risk of causing system overload. A simple distributed solution with minimal centralized control is random transmission decisions to meet an activity factor provided by the network. In this paper we propose uplink transmission timing, which aims at a provided activity factor, but carefully selects transmission instants to transmit when the channel is favorable. Simulations indicate that this scheme is more power-efficient in terms of power per kbps than random transmission and than ordinary dedicated channels with full activity. Furthermore, both the inter-cell interference and the transmission power are reduced significantly compared to random transmission decisions. The scheme also have the property of discontinuing the transmission if the channel conditions changes abruptly, which facilitates handover without critical interference problems during the transition phase.

\section{ACKNOWLEDGMENT}

This work is supported by the competence center ISIS (Information Systems for Industrial Control and Supervision) at Linköping University.

\section{REFERENCES}

[1] 3GPP, "Radio interface protocol architecture," Technical Specifi cation TSG RAN 25.301, 2003.

[2] — "Radio resource control (rrc) protocol specifi cation," Technical Specifi cation TSG RAN 25.331, 2003.

[3] C. Shannon, "The zero error capacity of a noisy channel," IRE Transactions On Information Theory, vol. 2, 1956.

[4] A. Goldsmith, "The capacity of downlink fading channels with variable rate and power," IEEE Transactions on Vehicular Technology, vol. 46, no. 3, 1997

[5] J. Schalkwijk, "Recent development in feedback communication," Proceedings of the IEEE, vol. 57, no. 7, 1969.

[6] A. Viterbi, A. Viterbi, and E. Zehavi, "Other-cell interference in cellular power-controlled CDMA," IEEE Transactions on Communications, vol. 42, no. 2, 1994.

[7] H. Holma and A. Toskala, Eds., WCDMA for UMTS. Radio Access for Third Generation Mobile Communications. Wiley, New York, NY, USA, 2000

[8] E. Geijer-Lundin, F. Gunnarsson, and F. Gustafsson, "Uplink load estimation in WCDMA," in Proc. IEEE Wireless Communications and Networking Conference, New Orleans, LA, USA, Mar 2003.

[9] F. Berggren, S.-L. Kim, R. Jæ ntti, and J. Zander, "Joint power control and intracell scheduling of DS-CDMA nonreal time data," IEEE Journal on Selected Areas in Communications, vol. 19, no. 10, Oct 2001.

[10] D. Rajan, A. Sabharwal, and B. Aazhang, "Impact of multiple access on uplink scheduling," in Proc. IEEE Information Theory Workshop, 2001.

[11] E. Villier, P. Legg, and S. Barrett, "Packet data transmissions in a WCDMA network-examples of uplink scheduling and performance," in Proc. IEEE Vehicular Technology Conference, Tokyo, Japan, May 2000.

[12] J. Stine and G. de Veciana, "Energy effi ciency of centrally controlled transmission of fi xed size packets," in Proc. IEEE Wireless Communications and Networking Conference, New Orleans, LA, USA, September 2000.

[13] A. Abrardo, G. Benelli, G. Giambene, and D. Sennati, "Performance analysis of a packet scheduling policy for a DS-CDMA cellular system," in Proc. IEEE Vehicular Technology Conference, 2001.

[14] S.-J. Oh, T. Lennon Olsen, and K. M. Wasserman, "Distributed power control and spreading gain allocation in CDMA data networks," in Proc. IEEE Infocom, 2000.

[15] S.-J. Oh and K. Wasserman, "Dynamic spreading gain control in multiservice CDMA networks," IEEE Journal on Selected Areas in Communications, vol. 17, no. 5, May 1999.

[16] S. Ramakrishna and J. Holtzman, "A scheme for throughput maximation in a dual-class CDMA system," IEEE Journal on Selected Areas in Communications, vol. 16, no. 6, August 1998.

[17] M. Yamada, Y. Hara, Y. Kamio, and S. Hara, "Packet communications with slotted ALOHA in a mobile cellular system," in Proc. IEEE Vehicular Technology Conference, Atlantic City, NJ, USA, October 2001.

[18] B. Sklar, "Rayleigh fading channels in mobile digital communication systems," IEEE Communications Magazine, vol. 35, no. 7, 1997.

[19] 3GPP, "Physical radio procedures," Technical Specifi cation TSG RAN 25.214, 2003.

[20] F. Gunnarsson, "Fundamental limitations of power control in WCDMA," in Proc. IEEE Vehicular Technology Conference, Atlantic City, NJ, USA, Oct 2001. 